\title{
Las instituciones municipales portuguesas y su adopción de las tecnologías 2.0: propuesta de un Indicador de Web Social
}

\author{
Valeriano Piñeiro-Naval* \\ Paulo Serra*
}

Artículo recibido:

26 de abril de 2018

Artículo aceptado:

15 de octubre de 2018

Artículo de investigación

\section{Resumen}

El propósito del estudio consiste en calibrar la adopción, a cargo de los municipios portugueses, de las tecnologías 2.0 como parte de su estrategia de comunicación digital. Para ello, y tras un análisis de contenido de los 308 sitios web del país, se creó un Indicador de Web Social (ICW) que, además de ofrecer una visión de conjunto, nos posibilitó identificar qué elementos, ya sean demográficos, económicos, geográficos, tecnológicos o políticos, afectan en mayor medida a esa adopción.

Los resultados indican que las redes sociales, especialmente Facebook, son el elemento más difundido, y que los factores clave en la implementación de las

* $\quad$ Facultad de Artes y Letras, Universidade da Beira Interior, Portugal

vale.naval@labcom.ubi.pt paulo.serra@labcom.ubi.pt

INVESTIGACIÓN BIBLIOTECOLÓGICA, vol.33, núm.78, enero/marzo, 2019, México, ISSN: 2448-8321 pp. 13-28 
herramientas 2.0 se vinculan, de forma significativa, con parámetros del desarrollo tecnológico de la localidad, su presupuesto y población. Estos factores apuntan al litoral y, más concretamente, al Área Metropolitana de Lisboa como el sector más destacado en cuanto al uso de la web colaborativa en sus pautas de e-Gobierno. A nivel ideológico, sin embargo, no existen diferencias entre ayuntamientos gobernados por partidos de izquierda o derecha en su consideración de las funcionalidades examinadas.

Palabras clave: Comunicación 2.0; Municipios; Análisis de Contenido; Indicador de Web Social

\title{
Portuguese municipal institutions and their adop-
} tion of 2.0 technologies: proposal of a Social Web Indicator

\author{
Valeriano Piñeiro-Naval and Paulo Serra
}

\section{Abstract}

The aim of the study is to measure the adoption, by Portuguese municipalities, of 2.0 technologies as part of their digital communication strategy. Therefore, and after making a content analysis of the 308 websites of the country, a Social Web Indicator (SWI) was created which, in addition to offering a vision of its whole, allowed us to identify those elements, whether demographical, economical, geographical, technological or political, affect to a greater extent this adoption.

The results indicate that social networks, especially Facebook, are the most widespread element; and that the key factors to implement the 2.0 tools, significantly relate to the parameters of technological development of the locality, as well as its budget and population. These factors point to the coast and, more specifically, to the Metropolitan Area of Lisbon as the geographical sector more highlighted, with regard to the use of Collaborative Web in its e-Government. At an ideological level, however, there are no differences among municipalities ruled by left or right parties in considering the examined functionalities.

Keywords: Communication 2.0; Municipalities; Content Analysis; Social Web Indicator 


\section{INTRODUCCIÓN}

T a importancia de las instituciones municipales en la vida de los ciudada-

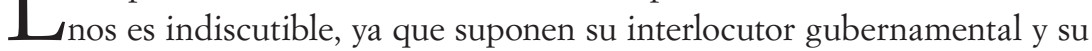
ente de representación política más inmediatos. En el presente contexto, "las sociedades y las organizaciones son permanentemente construidas por sus miembros a través de procesos comunicativos" (Johansson, 2007: 93); una circunstancia que no es ajena a la administración pública, donde el cambio de paradigma ha ocasionado, pese a su cariz burocratizado y parco en innovación, un fuerte impacto.

Según Vos (2009), las organizaciones gubernamentales han de cumplir con tres funciones a nivel comunicativo. En primer lugar, la comunicación corporativa (¿quién?) concibe la organización como un todo, incluyendo sus objetivos y la responsabilidad en su cumplimiento. En segundo lugar, la comunicación política (¿qué?) respalda las diversas áreas de actuación, como la educación, la economía, el transporte o el medio ambiente. Finalmente, la comunicación interna (¿cómo?) se ocupa de los procesos inherentes a la coordinación del organismo. Estas funciones deben ser implementadas mediante la utilización de lo que se ha dado en denominar e-Gobierno, una innovación capaz de mejorar procesos administrativos y gestiones técnicas a nivel institucional. En este sentido, el gobierno electrónico conlleva implícitamente un doble desafío: hacer más eficiente el sector público y establecer una relación horizontal entre ciudadanos y entidades.

El pilar sobre el que se sostiene el e-Gobierno es el formado por las TIC, que "prometen una transferencia de servicios más rápida, una mayor comunicación (por ejemplo, mediante la Web 2.0) y una plataforma más atractiva para el diálogo cívico" (Wang y Feeney, 2016: 297). Un elemento, la web colaborativa, resulta imprescindible para aumentar la interactividad con las audiencias y originar un mayor compromiso público, o public engagement (Rowe y Frewer, 2005). Entre las herramientas 2.0 se incluyen, sucintamente, "redes sociales como Facebook y Google+, servicios de microblogging como Twitter, blogs, wikis y sitios para compartir contenido multimedia como YouTube y Flickr" (Magro, 2012: 149).

Así, las redes sociales se perciben como instrumentos para gestionar estratégicamente las relaciones entre administraciones y públicos digitales (Lovari y Parisi, 2015), y no sólo mediante la transparencia o la participación, sino también a través de la promoción de la identidad propia y la divulgación de actos simbólicos (DePaula, Dincelli y Harrison, 2018). Del mismo modo, investigaciones precedentes señalan que el elemento de la Web 2.0 más extendido entre los municipios es el concerniente a las redes, especialmente 
Facebook (Agostino, 2013; Ellison y Hardey, 2014). Por ende, la primera hipótesis del estudio es:

H.1: el elemento de la Web 2.0 más extendido entre los municipios portugueses será el vinculado con las redes sociales.

Por tanto, el presente trabajo tiene un propósito fundamental: medir la utilización, a cargo de los municipios lusos, de las tecnologías 2.0 como parte de su estrategia de comunicación. Supeditado a este cometido, el segundo objetivo radica en identificar qué condicionantes (demográficos, económicos, geográficos, tecnológicos o políticos) afectan a esa utilización.

\section{Entes municipales en red: identificación de factores clave}

La bibliografía ocupada en identificar los factores externos (variables independientes) asociados al estado de los servicios electrónicos institucionales (variable dependiente) es cuantiosa. A continuación, referiremos algunos de esos factores que servirán de sustento para las restantes hipótesis.

Uno de los elementos de mayor consenso en la literatura especializada es el referido al número de habitantes (Graham, Avery y Park, 2015; Huang, 2006; Moon y Norris, 2005; Rufín, Medina y Sánchez-Figueroa, 2012). Por ello, trataremos de demostrar que

H.2: cuanto mayor sea la población de la localidad, mayor será la presencia de elementos de la Web 2.0 en su comunicación digital.

Tan destacada como la población, o incluso más, figura la riqueza de la localidad, otro parámetro clave en la implantación efectiva de prácticas e-gubernamentales (Gallego-Álvarez, Rodríguez-Domínguez y García-Sánchez, 2010; Gandía y Archidona, 2008; Gaule y Zilinskas, 2013; Holzer, Manoharan y Van Ryzin, 2010). Así pues, planteamos que

H.3: cuanto mayor sea la riqueza de la localidad, mayor será la presencia de elementos de la Web 2.0 en su estrategia de comunicación digital.

Desde el punto de vista de la ubicación geográfica, existen estudios que postulan la existencia de diferencias significativas entre regiones costeras e interiores, ya sea en el contexto de Portugal (Dias y Costa, 2013) o España (Piñeiro-Naval, Igartua y Marañón, 2017; Piñeiro-Naval, Igartua y Sánchez-Nuevo, 
H.4: la ubicación geográfica afectará a la implementación de los parámetros 2.0 en la estrategia de comunicación digital de los municipios, de modo que el litoral aventajará a los territorios del interior.

Los demás factores considerados en la literatura tienen que ver con el desarrollo tecnológico y con la ideología política del partido gobernante (Larsson, 2013), así como con el electorado (Ahn, 2011) y la transparencia municipal (Bonsón, Royo y Ratkai, 2015). Entonces, las demás hipótesis a verificar serían:

H.5: cuanto mayor sea el desarrollo tecnológico de la localidad, mayor será la presencia de elementos de la Web 2.0 en su estrategia de comunicación digital.

H.6: los municipios gobernados por partidos de izquierda tenderán a emplear, en mayor proporción, los parámetros de la Web 2.0 en comparación a los de derecha.

H.7: cuanto menor sea el porcentaje de electores entre la población local, mayor será la presencia de elementos de la Web 2.0 en la estrategia de comunicación digital del municipio.

H.8: cuanto mayor sea el nivel de transparencia municipal, mayor será la presencia de elementos de la Web 2.0 en la estrategia de comunicación digital de la localidad.

Se detalla acto seguido el método aplicado en la parte empírica del estudio.

\section{Metodología}

Como se ha avanzado anteriormente, el propósito del estudio consistió en elaborar un instrumento que permitiese ponderar el manejo de los rasgos de la comunicación 2.0 por parte de los municipios portugueses. Para ello, se diseñó un Indicador de Web Social que permitió efectuar una serie de comparaciones basadas en parámetros geográficos e ideológicos, así como correlacionarlo con otros de índole demográfica, económica o tecnológica; todo ello en aras de contrastar las hipótesis esbozadas.

Para el acometimiento de estos objetivos, se empleó el análisis de contenido por ser un método sistemático, objetivo y cuantitativo (Wimmer y Dominick, 2010), comúnmente aplicado al examen de entornos web (Herring, 2010). De partida, una de las consideraciones clave en cualquier análisis de contenido es el muestreo; y cuando el objeto de estudio es internet, la 
selección de muestras representativas supone todo un desafío para los estudiosos (Riffe, Lacy y Fico, 2014). Sin embargo, en esta investigación se recurrió al censo, ya que fue inspeccionado el universo de portales municipales portugueses: $N=308$ sitios web.

Como guía para el examen de este corpus, y fundamentada en estudios previos (Agostino, 2013; Bonsón, Royo y Ratkai, 2015; Fedotova, Teixeira y Alvelos, 2013; Larsson, 2013; Martínez-Rolán, Piñeiro-Otero y Baamonde-Silva, 2016; Roshan, Warren y Carr, 2016; Youngblood y Youngblood, 2018), fue configurada una checklist en la que se registró la presencia (valor "1") o la ausencia (valor "0") de enlaces efectivos, esto es, no huérfanos, a una serie de funcionalidades:

- Blogs (Google Blog, Blogger o WordPress).

- Micro-blogs (Twitter, Tumblr o Plurk).

- Boletín de noticias o newsletter.

- Redes sociales (Facebook o MySpace).

- Plataformas de imágenes (Instagram, Flickr o Picasa).

- Plataformas de video (YouTube o Vimeo).

- Foros, sugerencias o quejas.

- Y, finalmente, redes turísticas de recomendación (Tripadvisor o Booking).

En total, 8 variables dicotómicas, o dummy, a las que hay que añadir aquellas que sirvieron para identificar la unidad de análisis; a saber, el número del site municipal, el nombre de la localidad y la región NUTS $\mathrm{II}^{1}$ donde se enmarca. Del mismo modo, fueron recogidos datos estadísticos de cada municipio referentes a población, presupuesto, poder adquisitivo de sus habitantes, desarrollo tecnológico, partido de gobierno al frente del consistorio, electorado y transparencia municipal. ${ }^{2}$ Estos parámetros, que ejercieron como variables independientes, fueron cruzados con el Indicador de Web Social diseñado, tal como se comprobará en el apartado de resultados.

1 Las regiones NUTS (Nomenclatura de Unidades Territoriales para fines Estadísticos) II son un total de 7: las correspondientes al Portugal Continental (Norte, Centro, Alentejo, Área Metropolitana de Lisboa y Algarve), y las Regiones Autónomas Insulares de Azores y Madeira.

2 Las fuentes de información secundaria empleadas para recabar estos datos fueron las siguientes: Instituto Nacional de Estatística (censo poblacional, 2011: https://ine.pt/); Direção Geral das Autarquias Locais (presupuesto, 2017: https://www.portaldocidadao.pt/); Pordata. Base de dados de Portugal (poder adquisitivo de los ciudadanos, 2013: https://www.pordata.pt/); Secretaria-Geral do Ministério da Administração Interna (votantes en elecciones municipales 2013 y partidos de gobierno resultantes: https://www.sg.mai.gov.pt/), y Transparência e Integridade, Associação Cívica (Índice de Transparencia Municipal, 2017: https://transparencia. pt/). En el caso del Índice de Presença na Internet das Câmaras (IPIC), los datos para cada localidad fueron extraídos del informe publicado por Soares, Amaral y Ferreira (2017). El IPIC está estructurado a partir de 4 criterios: contenido; accesibilidad, navegabilidad y facilidad de uso; servicios online, y participación. 
En la recolección de los datos, que transcurrió desde el 16 de marzo hasta el 8 de junio de 2017, se vio implicado un equipo de dos codificadores. Tras este proceso, y para el chequeo de la fiabilidad, fue seleccionada una muestra aleatoria del $12 \%$ de los casos, que ambos evaluadores observaron en simultáneo. El parámetro estadístico utilizado para el cálculo de la fiabilidad fue el alfa de Krippendorff, hallado mediante el empleo de la macro Kalpha (Hayes y Krippendorff, 2007) para SPSS (versión 24). La fiabilidad media de las variables fue $\mathrm{M} \alpha_{\mathrm{k}}=0,90$ (Anexo).

\section{El Indicador de Web Social (IWS) de los municipios}

El elemento que ejerció de hilo conductor en la explotación de los datos fue el Indicador de Web Social (en adelante, IWS); un índice elaborado con la pretensión de medir, de forma agregada, el nivel de penetración de los municipios portugueses en la web social. La expresión alfanumérica que condensa la composición del IWS es:

$$
\text { IWS }=\sum\left(x_{1}, x_{2} \ldots x_{n}\right) / n
$$

donde: $x=$ variables $y n=$ número de variables.

Los ítems considerados para su diseño fueron los 4 que remitían más explícitamente a la Web 2.0; es decir, la tenencia de perfiles en redes sociales (Facebook o MySpace), micro-blogs (Twitter, Tumblr o Plurk), plataformas de video (YouTube o Vimeo) y, por último, plataformas de imágenes (Instagram, Flickr o Picasa).

Dado que estas categorías de análisis son dummy, el rango teórico del IWS, coincidente con su rango real, es " $0-1$ ". Los valores descriptivos para el conjunto de sitios web son $M_{\text {IWS }}=0,52(D T=0,30)$. Por otro lado, la consistencia interna del indicador asciende $\mathrm{a}_{\mathrm{c}}=0,64$; un valor admisible atendiendo al mínimo estipulado para estudios de carácter exploratorio: "0,60" (Hair et al., 2010; Robinson, Shaver y Wrightsman, 1991).

\section{Presentación y anÁlisis de Resultados}

En cuanto a las funcionalidades 2.0 respetadas por los municipios, pueden verse sus valores promedio en la Figura 1 . 


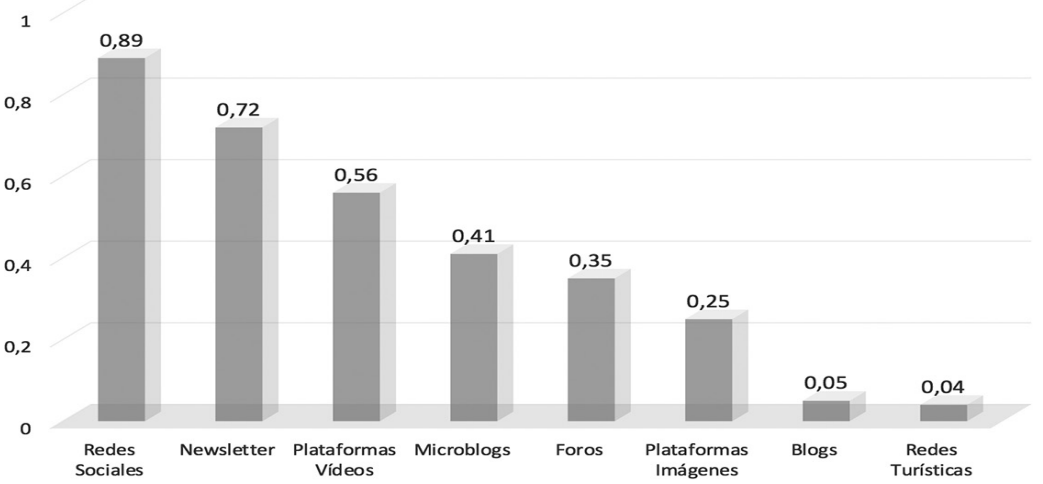

Figura 1. Valores promedio de los elementos 2.0 en la red local portuguesa

A tenor de estos datos, podemos afirmar que existen diferencias estadísticamente significativas entre los elementos $\left[\lambda_{\mathrm{w}}=0,115 ; F(7,301)=329,93 ; p<\right.$ 0,$\left.001 ; \eta^{2}=0,885\right]$. En este sentido, son las redes sociales $\left(H_{\cdot 1}\right)$ las que ostentan un mayor protagonismo $(M=0,89 ; D T=0,31)$, seguidas de los boletines de noticias $(M=0,72 ; D T=0,45)$ y las plataformas de video $(M=0,56 ; D T=0,49)$. De forma emparejada, se constatan, de nuevo, diferencias significativas entre las redes sociales y los boletines $[t(307)=5,43 ; p<0,001 ; d=0,44]$, así como entre los boletines y las plataformas de video $[t(307)=4,33 ; p<0,001$; $d=0,34]$; ambas diferencias, eso sí, "pequeñas" atendiendo al tamaño del efecto (Cohen, 1988).

Por otra parte, en la Tabla 1 pueden observarse los datos correspondientes a la asociación del IWS con distintos parámetros estadísticos (variables independientes):

\begin{tabular}{|c|c|c|c|}
\hline Parámetro & Fuente de información secundaria & $r$ & $\mathrm{~N}$ \\
\hline IPIC & Soares, Amaral y Ferreira (2017) & $0,273^{\star \star *}$ & 308 \\
\hline Presupuesto & Direção Geral das Autarquias Locais & $0,251^{\star \star \star}$ & 308 \\
\hline Población & Instituto Nacional de Estatística & $0,225^{\star \star \star}$ & 308 \\
\hline Electorado & Secretaria-Geral do Ministério da Administração Interna & $-0,172^{\star \star}$ & 308 \\
\hline Poder adquisitivo & Pordata. Base de dados de Portugal & $0,158^{\star \star}$ & 308 \\
\hline ITM & Transparência e Integridade, Associação Cívica & $0,139^{*}$ & 308 \\
\hline
\end{tabular}

Tabla 1. Asociación entre los parámetros estadísticos de los municipios y el IWS ( $r$ de Pearson)

$$
\text { Nota: }{ }^{*} \leq 0,05 ;{ }^{* *} p \leq 0,01 ;{ }^{* * *} p \leq 0,001
$$


Se observa que el IWS correlaciona muy significativamente con el parámetro de desarrollo tecnológico tenido en cuenta; esto es, el IPIC $[r(306)=0,273$; $p$ $<0,001]\left(\mathrm{H}_{.5}\right)$, bien como con el presupuesto municipal $[r(306)=0,251 ; p<$ $0,001]\left(\mathrm{H}_{2}\right)$ y el número de habitantes $\left.[r(306)=0,225 ; p<0,001]\left(\mathrm{H}_{\cdot}\right)\right)$. Del mismo modo, también se asocia significativamente con el poder adquisitivo de los ciudadanos $[r(306)=0,158 ; p=0,005]\left(\mathrm{H}_{2}\right)$ y el índice de transparencia, o ITM $[r(306)=0,139 ; p=0,014]\left(\mathrm{H}_{8}\right)$. Finalmente, el IWS correlaciona significativamente, aunque en sentido negativo, con el electorado $[r(306)=$ $-0,172 ; p=0,002]\left(\mathrm{H}_{.}\right)$, de modo que, a medida que el electorado disminuye, el IWS aumenta (y viceversa).

En relación con la localización geográfica de los municipios $\left(\mathrm{H}_{4}\right)$, la Tabla 2 muestra las medias del IWS según las regiones NUTS II:

\begin{tabular}{|l|c|c|c|}
\hline \multicolumn{1}{|c|}{ Regiones NUTS II } & $\mathbf{M}_{\text {IWS }}$ & DT & $\mathbf{n}$ \\
\hline Norte & 0,59 & 0,31 & 86 \\
\hline Centro & 0,46 & 0,29 & 100 \\
\hline Alentejo & 0,46 & 0,26 & 58 \\
\hline Área Metropolitana de Lisboa & $0,77 a$ & 0,30 & 18 \\
\hline Algarve & 0,57 & 0,28 & 16 \\
\hline Azores & $0,38 \mathrm{a}$ & 0,29 & 19 \\
\hline Madeira & 0,63 & 0,28 & 11 \\
\hline Total & $\mathbf{0 , 5 2}$ & $\mathbf{0 , 3 0}$ & $\mathbf{3 0 8}$ \\
\hline
\end{tabular}

Tabla 2. Promedio del IWS con arreglo de las regiones NUTS II (ANOVA)

Nota: las medias acompañadas de la letra subíndice "a"

se diferencian entre sí muy significativamente $(p<0,001)$

Después de practicar un análisis de la varianza, es posible ratificar el desequilibrio que se establece entre las regiones NUTS II en cuanto a la implementación de las prácticas digitales 2.0 en sus municipios $\left[F_{\text {IWS x NUTS II }}(6,301)=5,039\right.$; $\left.p<0,001 ; \lambda^{2}=0,091\right]$. A este nivel, y tras la prueba post hoc T3 de Dunnett, se constata que las mayores diferencias son las que se generan entre el Área Metropolitana de Lisboa y las Islas Azores [ $t(35)=4,011 ; p<0,001 ; d=1,32]$; unos desajustes "elevados" según el tamaño del efecto (Cohen, 1988).

Si nos centramos ahora en el criterio geográfico "litoral frente interior", los valores del IWS para ambos sectores son los siguientes (Figura 2): 


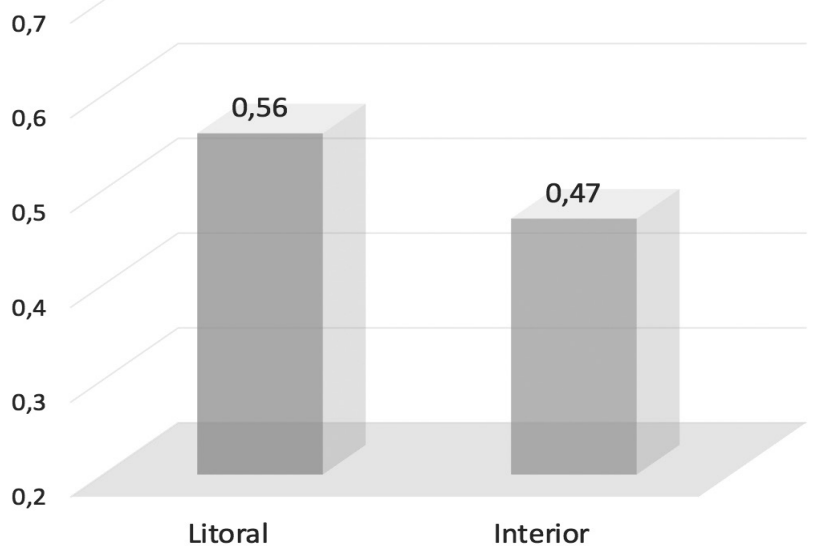

Figura 2. Valores promedio del IWS según el cotejo "litoral frente interior"

Según los datos visualizados en la Figura 2, el litoral del país $(M=0,56$; $D T=0,30)$ suele hacer un uso más intensivo de la comunicación 2.0 en comparación con los territorios del interior $(M=0,47 ; D T=0,30)$; y de una forma, además, estadísticamente significativa $[t(306)=2,56 ; p<0,001 ; d=0,30]$.

Para finalizar, y en términos políticos, se procedió a efectuar una nueva comparación intermunicipal cimentada, ahora, en la disyuntiva "izquierda frente derecha". Tras el cálculo de la prueba $t$ de Student, las divergencias entre el IWS según los municipios regidos por partidos de izquierda $(M=0,52$; $D T=0,30)$ y de derecha $(M=0,53 ; D T=0,31)$ son completamente inexistentes $[t(306)=-0,094 ; p=0,925 ; d=-0,032]\left(\mathrm{H}_{6}\right)$.

\section{Discusión}

Estos resultados nos permiten establecer paralelismos con otros estudios, de igual propósito y avanzados en la introducción, derivados de investigaciones internacionales previas. Así pues, Agostino (2013) llega a la conclusión, tras

3 Para clasificar los municipios en función de la ideología de su partido de gobierno, se procedió a la recodificación de la variable polinómica "partido político" (extraída a partir de los datos de la Secretaria-Geral do Ministério da Administração Interna) en otra dicotómica llamada "ideología de gobierno", cuyos valores son 1 = Izquierda y 2 = Derecha. Se incluyeron en el ala izquierda al Partido Socialista, Partido Comunista y Partido Ecologista, y a los Grupos de Ciudadanos independientes; en el ala derecha fueron considerados el Partido Social Demócrata y el Partido Popular. 
un análisis de 119 portales municipales italianos, que Facebook es la herramienta social más empleada (92\%); un dato no demasiado alejado de $68 \%$ al que apuntan Ellison y Hardey (2014) tras examinar 352 sitios británicos. En el caso portugués, supone $89 \%$.

En cuanto al factor poblacional, Graham, Avery y Park (2015) determinan que cuanto mayor sea el número de habitantes de la localidad, más notable será la adopción de estrategias 2.0 por parte, en su caso, de una muestra de 300 municipios de los Estados Unidos. Estos resultados son congruentes con nuestro trabajo y otros precedentes, como los de Moon y Norris (2005) o Huang (2006), quienes sostienen que la población juega un papel fundamental en la puesta en marcha del e-Gobierno.

La riqueza, por su parte, se erige en otro de los grandes condicionantes del estado de la web local. Así lo subrayan estudios a nivel comparativo mundial, como los de Gallego-Álvarez, Rodríguez-Domínguez y García-Sánchez (2010) o también Holzer, Manoharan y Van Ryzin (2010). En el contexto español, Gandía y Archidona (2008) examinan, a nivel informativo, los portales de 130 ayuntamientos superiores a 50000 habitantes; mientras que Gaule y Zilinskas (2013) testan la calidad web de 60 websites de Lituania. En todos se llega a la misma conclusión: la riqueza desempeña un rol sustancial en la implementación de servicios online.

A nivel de ubicación geográfica, Piñeiro-Naval, Igartua y Marañón (2017) señalan, a partir de una muestra de 500 sedes, la existencia de diferencias estadísticamente significativas entre la calidad del diseño web de los municipios del litoral y del interior de España. Una circunstancia verificada, asimismo, por Dias y Costa (2013) en Portugal, esta vez con relación a las prácticas de gobierno electrónico.

Con respecto al desarrollo tecnológico, Larsson (2013) postula, fundamentándose en una muestra de 290 portales suecos, que cuanto mayor sea el porcentaje de ciudadanos con acceso a servicios web de banda ancha, más intensivo será el empleo de los medios sociales por parte de los municipios. Aunque en nuestro caso no se empleó ese indicador, sino el IPIC, se verifica que la presencia (general) en internet de los concejos correlaciona con el manejo de tecnologías (específicas) 2.0. Este mismo autor plantea igualmente que los partidos de izquierda se sirven en mayor medida de los medios sociales frente a los conservadores. En el contexto de Portugal no se corrobora tal circunstancia; esto es, no existen contrastes entre unos partidos y otros en la consideración que le otorgan a la web colaborativa.

Sin alejarnos del terreno político, Ahn (2011) llega a la conclusión, a partir de una muestra de 857 sitios municipales norteamericanos, que cuanto menor es el electorado en estas localidades mayor es el uso que los gestores 
gubernamentales hacen de los social media. Esta hipótesis sí pudo verificarse en el contexto luso.

Por último, Bonsón, Royo y Ratkai (2015) afirman que el public engagement con las redes sociales por parte de los ciudadanos europeos (se valen de una muestra de 75 ciudades de todo el continente) aumenta a medida que la transparencia de los municipios crece. En Portugal se produce una circunstancia parecida: cuanta mayor transparencia, mayor propensión a la utilización de la web social a nivel municipal.

En resumen, la Tabla 3 refleja las hipótesis del estudio, verificadas en su mayoría:

\begin{tabular}{|c|c|}
\hline $\mathbf{N}^{0}$ Hipótesis & Resolución \\
\hline$H_{.1}$ & $\sqrt{ }$ \\
\hline$H_{._{2}}$ & $\sqrt{ }$ \\
\hline$H_{._{3}}$ & $\sqrt{ }$ \\
\hline$H_{._{4}}$ & $\sqrt{ }$ \\
\hline$H_{._{5}}$ & $X$ \\
\hline$H_{6_{6}}$ & $\sqrt{ }$ \\
\hline$H_{._{7}}$ & $\sqrt{ }$ \\
\hline$H_{.8}$ & \\
\hline
\end{tabular}

Tabla 3. Relación de hipótesis del estudio Nota: $\sqrt{ }=$ confirmada; $X=$ refutada.

\section{CONCLUSIONES Y RECOMENDACIONES}

La meta del estudio radicó en calibrar el manejo, por parte de los ayuntamientos portugueses, de las tecnologías 2.0 como uno de los recursos de su estrategia de comunicación institucional. Para ello, y tras un análisis de contenido de los 308 sitios web oficiales del país, se creó un Indicador de Web Social (ICW) que, además de ofrecer una panorámica general de su conjunto, nos posibilitó identificar qué elementos, ya fuesen demográficos, económicos, geográficos, tecnológicos o políticos, afectaban en mayor medida a esa adopción.

Por tanto, sabemos que las redes sociales (cuyo máximo exponente es Facebook) son el elemento más extendido, seguido de los boletines de noticias y las plataformas de video, donde YouTube acapara el mayor protagonismo. Debemos tener en cuenta que, pese a la irrupción constante de nuevos medios sociales (Instagram parece ser ahora el que acapara todos los focos), la red ideada por Mark Zuckerberg sigue estando muy vigente debido al histórico 
arraigo que ya manifiesta, a nivel mundial, entre sus usuarios, ya sean organizaciones o individuos.

Por otro lado, hemos comprobado que los factores de desarrollo tecnológico, presupuestarios y demográficos presentan una incidencia determinante en el nivel de implantación de la web social a cargo de los organismos municipales. En Portugal, país que nos ocupa, las localidades más desarrolladas, ricas y pobladas están en el litoral; un hecho que se refuerza a partir de las comparaciones geográficas efectuadas. Consecuentemente, tanto la administración estatal como los propios municipios del interior deberán tratar de paliar la brecha digital que los separa de los costeros si quieren avanzar, al mismo ritmo, en la implantación homogénea del e-Gobierno en todo el territorio.

En suma, las ciudades, como cualquier otro tipo de organización, desean generar imágenes y asociaciones positivas (Zavattaro, 2010) entre sus conciudadanos y transmitirles debidamente quiénes son, qué hacen y cómo; un propósito al que puede contribuir un empleo estratégico de las herramientas 2.0, destinadas a reorientar la comunicación entre las instituciones y los usuarios a un plano horizontal.

\section{Agradecimientos}

Este trabajo parte de un proyecto de investigación financiado por la Fundação para a Ciência e a Tecnologia (Ref.: SFRH/BPD/122402/2016) de Portugal.

\section{REFERENCIAS}

Agostino, Deborah. 2013. "Using social media to engage citizens: A study of Italian municipalities”. Public Relations Review 39: 232-234. doi: http://dx.doi.org/10.1016/j.pubrev.2013.02.009

Ahn, Michael J. 2011. "Adoption of E-Communication Applications in U.S. Municipalities: The Role of Political Environment, Bureaucratic Structure, and the Nature of Applications". The American Review of Public Administration 41 (4): 428452. doi: http://dx.doi.org/10.1177/0275074010377654

Bonsón, Enrique, Sonia Royo y Melinda Ratkai. 2015. “Citizens' engagement on local governments' Facebook sites. An empirical analysis: The impact of different media and content types in Western Europe”. Government Information Quarterly 32: 52-62. doi: http://dx.doi.org/10.1016/j.giq.2014.11.001

Cohen, Jacob. 1988. Statistical power analysis for the behavioral sciences, 2a. ed. Hillsdale, New Jersey: Lawrence Earlbaum Associates. 
DePaula, Nic, Ersin Dincelli y Teresa M. Harrison. 2018. “Toward a typology of government social media communication: Democratic goals, symbolic acts and self-presentation”. Government Information Quarterly 35: 98-108. doi: http://dx. doi.org/10.1016/j.giq.2017.10.003

Dias, Gonçalo Paiva y Marco Costa. 2013. "Significant socio-economic factors for local e-government development in Portugal”. Electronic Government, An International Journal 10 (3-4): 284-309. doi: http://dx.doi.org/10.1504/EG.2013.058785

Ellison, Nick y Michael Hardey. 2014. "Social Media and Local Government: Citizenship, Consumption and Democracy”. Local Government Studies 40 (1): 21-40. doi: http://dx.doi.org/10.1080/03003930.2013.799066

Fedotova, Olga, Leonor Teixeira y Helena Alvelos. 2013. "E-participation in Portuguese local governments: evaluation of deployment and adherence of Web 2.0 technologies”. Electronic Government, An International Journal 10 (3-4): 361-379. doi: http://dx.doi.org/10.1504/EG.2013.058789

Gallego-Álvarez, Isabel, Luis Rodríguez-Domínguez e Isabel-María García-Sánchez. 2010. "Are determining factors of municipal E-government common to a worldwide municipal view? An intra-country comparison". Government Information Quarterly 27: 423-430. doi: http://dx.doi.org/10.1016/j.giq.2009.12.011

Gandía, Juan L. y Maria C. Archidona. 2008. "Determinants of web site information by Spanish city councils”. Online Information Review 32 (1): 35-57. doi: http://dx. doi.org/10.1108/14684520810865976

Gaule, Egle y Gintaras Zilinskas. 2013. "E-governance in Lithuanian Municipalities: External Factors Analysis of the Websites Development”. Public Policy and Administration 12 (1): 80-93. doi: http://dx.doi.org/10.5755/j01.ppaa.12.1.3854

Graham, Melissa W., Elizabeth J. Avery y Sejin Park. 2015. "The role of social media in local government crisis communications". Public Relations Review 41: 386-394. doi: http://dx.doi.org/10.1016/j.pubrev.2015.02.001

Hair, Joseph F., William C. Black, Barry J. Babin y Rolph E. Anderson. 2010. Multivariate data analysis, 7a. ed. Upper Saddle River, New Jersey: Pearson Prentice Hall.

Hayes, Andrew F. y Klaus Krippendorff. 2007. "Answering the call for a standard reliability measure for coding data". Communication methods and measures 1 (1): 77-89. doi: http://dx.doi.org/10.1080/19312450709336664

Herring, Susan C. 2010. "Web Content Analysis: Expanding the Paradigm”, en International Handbook of Internet Research, editado por Jeremy Hunsinger, Lisbeth Klastrup y Matthew Allen, 233-249. Springer Netherlands.

Holzer, Marc, Aroon Manoharan y Gregg Van Ryzin. 2010. "Global cities on the Web: An empirical typology of municipal websites". International Public Management Review 11 (3): 104-121.

Huang, Zhenyu. 2006. "E-Government practices at local levels: an analysis of U.S. counties' websites". Issues in Information Systems 7 (2): 165-170.

Johansson, Catrin. 2007. "Research on Organizational Communication. The Case of Sweden”. Nordicom Review 28 (1): 93-110. doi: http://dx.doi.org/10.1515/ nor-2017-0203

Larsson, Anders Olof. 2013. "Bringing it all back home? Social media practices by Swedish municipalities”. European Journal of Communication 28 (6): 681-695. doi: http://dx.doi.org/10.1177/0267323113502277 
Lovari, Alessandro y Lorenza Parisi. 2015. "Listening to digital publics. Investigating citizens' voices and engagement within Italian municipalities' Facebook Pages”. Public Relations Review 41: 205-213. doi: http://dx.doi.org/10.1016/j.pubrev.2014.11.013

Magro, Michael J. 2012. "A Review of Social Media Use in E-Government". Administrative Sciences 2: 148-161. doi: http://dx.doi.org/10.3390/admsci2020148

Martínez-Rolán, Xabier, Teresa Piñeiro-Otero y Xosé Manuel Baamonde-Silva. 2016. "El desafío de la transparencia en la comunicación digital. Un estudio de caso sobre las webs municipales de Galicia y Norte de Portugal”. Observatorio $\left(O B S^{*}\right) 10(4): 35-55$.

Moon, M. Jae y Donald F. Norris. 2005. "Does managerial orientation matter? The adoption of reinventing government and e government at the municipal level". Information Systems Journal 15 (1): 43-60. doi: http://dx.doi.org/10.1111/j.1365-2575.2005.00185.x

Piñeiro-Naval, Valeriano, Juan José Igartua y Felipe Marañón. 2017. “El diseño de las sedes web municipales de España. Una propuesta metodológica para su análisis”. Revista Española de Documentación Científica 40 (1): e164. doi: http://dx. doi.org/10.3989/redc.2017.1.1368

Piñeiro-Naval, Valeriano, Juan José Igartua y Lucía-Alejandra Sánchez-Nuevo. 2017. "Identificación de factores externos que predicen el estado de los sitios web municipales: una aproximación regional al caso de España”. Observatorio (OBS*) 11 (3): 44-60.

Riffe, Daniel, Stephen Lacy y Frederick Fico. 2014. Analyzing Media Messages. Using Quantitative Content Analysis in Research, 3a. ed. New York: Routledge.

Robinson, John P., Phillip R. Shaver y Lawrence S. Wrightsman. 1991. "Criteria for scale selection and evaluation", en Measures of personality and social psychological attitudes, editado por John P. Robinson, Phillip R. Shaver y Lawrence S. Wrightsman, 1-16. San Diego: Academic Press.

Roshan, Mina, Matthew Warren y Rodney Carr. 2016. "Understanding the use of social media by organisations for crisis communication". Computers in Human Behaviour 63: 350-361. doi: http://dx.doi.org/10.1016/j.chb.2016.05.016

Rowe, Gene y Lynn J. Frewer. 2005. "A typology of public engagement mechanisms". Science, Technologies and Human Values 30 (2): 251-290. doi: http://dx.doi. org/10.1177/0162243904271724

Rufín, Ramón, Cayetano Medina y Juan-Carlos Sánchez-Figueroa. 2012. "Moderating Factors in Adopting Local e-Government in Spain”. Local Government Studies 38 (3): 367-385. doi: http://dx.doi.org/10.1080/03003930.2011.636035

Soares, Delfina Sá, Luís Amaral y Luis Miguel Ferreira. 2017. Presença na Internet das Câmaras Municipais portuguesas em 2016: Estudo sobre Local e-Government em Portugal. Universidade do Minho, Guimarães: Gávea - Observatório da Sociedade da Informação.

Vos, Marita. 2009. "Communication quality and added value: a measurement instrument for municipalities”. Journal of Communication Management 13 (4): 362-377. doi: http://dx.doi.org/10.1108/13632540911004623

Wang, Shu y Mary K. Feeney. 2016. "Determinants of Information and Communication Technology Adoption in Municipalities". American Review of Public Administration 46 (3): 292-313. doi: http://dx.doi.org/10.1177/0275074014553462 
Wimmer, Roger D. y Joseph R. Dominick. 2010. Mass Media Research: An Introduction, 9a. ed. Boston: Wadsworth.

Youngblood, Susan A. y Norman E. Youngblood. 2018. "Usability, content, and connections: How county-level Alabama emergency management agencies communicate with their online public". Government Information Quarterly 35: 50-60. doi: http://dx.doi.org/10.1016/j.giq.2017.12.001

Zavattaro, Staci M. 2010. "Municipalities as Public Relations and Marketing Firms". Administrative Theory E Praxis 32 (2): 191-211. doi: http://dx.doi.org/10.2753/ ATP1084-1806320202

\begin{tabular}{|c|c|}
\hline Variables de la checklist & Alfa de Krippendorff $\left(\alpha_{k}\right)$ \\
\hline 1. Blogs: Google Blog, Blogger o WordPress & 1 \\
\hline 2. Micro-blogs: Twitter, Tumblr o Plurk & 0,88 \\
\hline 3. Boletín de noticias o newsletter & 0,83 \\
\hline 4. Redes sociales: Facebook o MySpace & 0,87 \\
\hline 5. Plataformas de imágenes: Instagram, Flickr o Picasa & 0,92 \\
\hline 6. Plataformas de video: YouTube o Vimeo & 0,95 \\
\hline 7. Foros, sugerencias o quejas & 0,73 \\
\hline 8. Redes turísticas de recomendación: Tripadvisor o Booking & 1 \\
\hline Total & 0,90 \\
\hline
\end{tabular}

Para citar este texto:

Piñeiro-Naval, Valeriano y Paulo Serra. 2019. "Las instituciones municipales portuguesas y su adopción de las tecnologías 2.0: propuesta de un Indicador de Web Social”. Investigación Bibliotecológica: archivonomía, bibliotecología e información 33 (78): 13-28.

http://dx.doi.org/10.22201/iibi.24488321xe.2019.78.57957 